\title{
Sufficient conditions for oscillations in higher order linear functional differential equations of neutral type
}

\author{
By Jaroslav JARoš and Takaŝi KusANo
}

(Received September 14, 1988)

\section{$\S 1$. Introduction}

Our main aim in this paper is to derive oscillation criteria for neutral functional differential equations of the form

$$
\frac{d^{n}}{d t^{n}}[x(t)+\lambda x(t+\rho)]+\sigma \sum_{i \in I} p_{i} x\left(t+\tau_{i}\right)=0, \quad t \geqq t_{0},
$$

where $\sigma=+1$ or $-1, I$ is an initial segment of natural numbers, $p_{i}, i \in I$, are positive constants and $\lambda, \rho$ and $\tau_{i}, i \in I$, are real numbers.

The literature on oscillation of solutions of neutral functional differential equations is growing day by day. We refer, in particular, to the papers of Grammatikopoulos, Grove and Ladas [2-4], Grammatikopoulos, Ladas an Meimaridou [5], Grammatikopoulos, Ladas and Sficas [6], Grove, Kulenović and Ladas [7], Grove, Ladas and Meimaridou [8], Ivanov and Kusano [9], the present authors [10], Kulenović, Ladas and Meimaridou [12], Ladas and Sficas [14], Ruan [19], Sficas and Stavroulakis [20] and Zahariev and Bainov [21-22]. Most of the papers mentioned above, however, concerns the first and second order equations. Our study here is related to the recent work of Ladas and Sficas [15] which is one of the first attempts in a systematic investigation of oscillatory properties of higher order neutral equations.

The outline of the present paper is the following.

Section 2 is devoted to a study of asymptotic and oscillatory properties of "ordinary" functional differential inequalities with several retarded and advanced arguments. In Section 3 we classify the possible nonoscillatory solutions of Eq. (E, $\sigma$ ) according to their asymptotic behavior. In order to preclude all the solution classes appearing in this classification (i.e. to ensure the oscillation of all solutions), we derive some "ordinary" functional differential inequalities which have nonoscillatory solutions with specific asymptotic growth provided that $(\mathrm{E}, \sigma)$ has solutions of the similar kind. Finally, in Section 4 we apply the results of the preceding sections to these inequalities and derive oscillation criteria for the original neutral equation 
$(\mathrm{E}, \sigma)$

In what follows, by a soultion of $(\mathrm{E}, \sigma)$ we mean a continuous function $x(t)$ defined on an interval $\left[t_{x}, \infty\right) t_{x} \geqq t_{0}$, which satisfies (E, $\sigma$ ) (so that $x(t)+$ $\lambda x(t+\rho)$ is $n$-times continuously differentiable) for all sufficiently large $t$. The oscillatory character of the solution $x(t)$ of $(\mathrm{E}, \sigma)$ is considered in the usual sense, that is, $x(t)$ is said to be oscillatory if it has arbitrarily large zeros in $\left[t_{x}, \infty\right)$ and it is said to be nonoscillatory otherwise.

\section{$\S 2$. Functional differential inequalities of retarded, advanced and mixed type}

Consider the linear functional differential inequalities of the form

$(\mathrm{M}, \sigma)$

$$
\left\{\sigma x^{(n)}(t)+\sum_{i \in I} p_{i} x\left(t+\tau_{i}\right)\right\} \operatorname{sgn} x(t) \leqq 0, \quad t \geqq t_{0},
$$

where $\sigma=+1$ or $-1, I$ is an initial segment of natural numbers, $p_{i}, i \in I$, are positive constants and $\tau_{i}, i \in I$, are real numbers.

It is a standard result that if $u \in C^{n}\left(\left[t_{0}, \infty\right), \boldsymbol{R}\right)$ is such that

$$
u(t) \neq 0 \text { and } \sigma u(t) u^{(n)}(t)<0 \text { for } t \geqq t_{0},
$$

then there exist an integer $l \in\{0,1, \cdots, n\}$ and a $t_{1} \geqq t_{0}$ such that $(-1)^{n-l-1} \sigma=1$ and

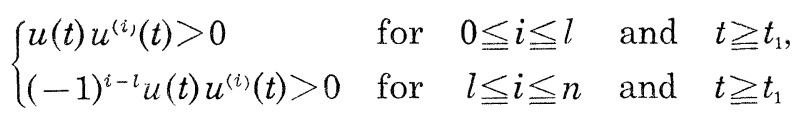

(see, for example, Kiguradze [11]).

A function $u(t)$ satisfying $(2.2)_{l}$ is termed a function of (Kiguradze) degree $l$.

Clearly, if $x(t)$ is a nonoscillatory solution of inequality $(\mathrm{M}, \sigma)$, then it satisfies (2.1) for all large $t$, and so $x(t)$ is a function of Kiguradze degree $l$ for some $l \in\{0,1, \cdots, n\}$ with $(-1)^{n-l-1} \sigma=1$.

Denote by $\mathscr{N}_{l}$ the set of all solutions of $(M, \sigma)$ which are of degree $l$. Then it is not difficult to see that $\mathscr{N}_{l}=\phi$ for $0<l<n$. In fact, if $x(t)$ is a solution of degree $l, 0<l<n$, say positive, then $x(t)$ is an eventually increasing function, and so there exist a positive constant $c$ and a $t_{1} \geqq t_{0}$ such that $x\left(t+\tau_{i}\right) \geqq c$ for every $i \in I$ and $t \geqq t_{1}$. Then we get from $(\mathrm{M}, \sigma)$ that

$$
x^{(n)}(t) \leqq-c \sum_{i \in I} p_{i}<0, \quad t \geqq t_{1}, \quad \text { for } \sigma=+1
$$

and

$$
x^{(n)}(t) \geqq c \sum_{i \in I} p_{i}>0, \quad t \geqq t_{1}, \quad \text { for } \sigma=-1,
$$


which implies that

$$
\lim _{t \rightarrow \infty} x^{(i)}(t)=-\infty, \quad i=0,1, \cdots, n-1 \quad \text { for } \sigma=+1
$$

and

$$
\lim _{t \rightarrow \infty} x^{(i)}(t)=\infty, \quad i=0,1, \cdots, n-1 \quad \text { for } \sigma=-1 .
$$

The first conclusion contradicts the positivity of $x(t)$, while the second one gives a contradiction with the decreasing character of $x^{(l)}(t)$ for $x \in \mathscr{N}_{l}$, $0<l<n$. In an analogous way we are led to a contradiction also in the case of an eventually negative $x(t)$.

Consequently, the set $\mathscr{N}$ of all possible nonoscillatory solutions of $(\mathrm{M}, \sigma)$ has the following decomposition:

$$
\begin{array}{ll}
\mathscr{N}=\phi & \text { for }(\mathrm{M},+1) \text { with } n \text { even, } \\
\mathscr{N}=\mathscr{N}_{0} & \text { for }(\mathrm{M},+1) \text { with } n \text { odd, } \\
\mathscr{N}=\mathscr{N}_{0} \cup \mathscr{N}_{n} & \text { for }(\mathrm{M},-1) \text { with } n \text { even, } \\
\mathscr{N}=\mathscr{N}_{n} & \text { for }(\mathrm{M},-1) \text { with } n \text { odd. }
\end{array}
$$

Denote

$$
\begin{array}{ll}
R_{0}=\left\{i \in I: \tau_{i} \leqq 0\right\}, & R=\left\{i \in I: \tau_{i}<0\right\}, \\
A_{0}=\left\{i \in I: \tau_{i} \geqq 0\right\}, & A=\left\{i \in I: \tau_{i}>0\right\},
\end{array}
$$

and assume first that either $I=R_{0}$ or $I=A_{0}$.

Analysing the characteristic equations of the functional differential equations corresponding to $(\mathrm{M},+1)$ and $(\mathrm{M},-1)$, we easily see that the subclasses $\mathscr{N}_{0}$ and $\mathscr{N}_{n}$ are always nonempty for $(\mathrm{M},+1)$ with $n$ odd and $I=A_{0}$ and for $(\mathrm{M},-1)$ with $n$ odd and $I=R_{0}$, respectively. In other cases, however, $\mathscr{N}_{0}$ or $\mathscr{N}_{n}$ may be excluded from the above classification provided that certain conditions are satisfied.

The following theorem gives a characterization of such situation.

TheOREM 2.1. (i) Let $I=R_{0}$. Then

$$
\mathscr{N}=\phi \quad \text { for }(\mathrm{M},+1) \text { with } n \text { odd }
$$

and

$$
\mathscr{N}=\mathscr{N}_{n} \text { for }(\mathrm{M},-1) \text { with } n \text { even }
$$

if and only if

$$
\alpha^{n}-\sum_{i \in R_{0}} p_{i} e^{-\alpha \tau_{i}}<0
$$


for all $\alpha>0$

(ii) Let $I=A_{0}$. Then

$$
\mathscr{N}=\mathscr{N}_{0} \quad \text { for }(\mathrm{M},-1) \text { with } n \text { even }
$$

and

$$
\mathscr{N}=\phi \quad \text { for }(\mathrm{M},-1) \text { with } n \text { odd }
$$

if and only if

$$
\alpha^{n}-\sum_{i \in A_{0}} p_{i} e^{\alpha \tau i}<0
$$

for all $\alpha>0$.

This theorem is essentially due to Ladas, Sficas and Stavroulakis [16]. Although they have proved it only for the corresponding equations, it is not difficult to verify with the aid of the comparison principle of Philos [18] and an analogous result for advanced equations that it remains true also for the inequalities under consideration. For an alternative proof of its sufficiency part in the case (i) see also Čanturia [1].

Since, in general, it is not an easy matter to check the validity of (2.3) (or (2.4)) directly, we now look for some explicit sufficient conditions which ensure that (2.3) (or (2.4)) is satisfied.

Theorem 2.2. (i) Let $I=R_{0}$. Then each of the following conditions implies that (2.3) is satisfied:

(a) $\sum_{i \in R} p_{i}\left(-\tau_{i}\right)^{n}>(n / e)^{n}$,

(b) $\left|R_{0}\right|^{1-n}\left(\sum_{i \in R}\left(-\tau_{i}\right)\right)^{n}\left(\prod_{i \in R_{0}} p_{i}\right)^{1 /\left|R_{0}\right|}>(n / e)^{n}$,

where $\left|R_{0}\right|$ denotes the number of the members of $R_{0}$.

(ii) Let $I=A_{0}$. Then each of the following conditions implies that (2.4) is satisfied:

(c) $\sum_{i \in A} p_{i} \tau_{i}^{n}>(n / e)^{n}$,

(d) $\left|A_{0}\right|^{1-n}\left(\sum_{i \in A} \tau_{i}\right)^{n}\left(\prod_{i \in A_{0}} p_{i}\right)^{1 /\left|A_{0}\right|}>(n / e)^{n}$,

where $\left|A_{0}\right|$ denotes the number of the members of $A_{0}$.

Proof. Assume first $I=R_{0}$ and consider the function

$$
F(\alpha)=1-\sum_{i \in R_{0}} p_{i} e^{-\alpha \tau_{i}} \alpha^{-n}, \quad \alpha>0 .
$$

Let (a) be satisfied. Clearly, 


$$
F(\alpha) \leqq 1-\sum_{i \in R} p_{i} e^{-\alpha \tau i} \alpha^{-n} \quad \text { for } \alpha>0
$$

and minimizing every expression $p_{i} e^{-\alpha \tau} \alpha^{-n}, i \in R$, by setting $\alpha=-n / \tau_{i}$ we find

$$
F(\alpha) \leqq 1-(e / n)^{n} \sum_{i \in R} p_{i}\left(-\tau_{i}\right)^{n}<0
$$

for all $\alpha>0$. Consequently,

$$
\alpha^{n} F(\alpha)<0
$$

for all $\alpha>0$, which is nothing else than (2.3).

In proving (b) we first use the arithmetic mean-geometric mean inequality

$$
\begin{aligned}
F(\alpha) & \leqq 1-\left|R_{0}\right|\left(\prod_{i \in R_{0}} p_{i} e^{-\alpha \tau_{i}} \alpha^{-n}\right)^{1 /\left|R_{0}\right|} \\
& =1-\left|R_{0}\right|\left(\prod_{i \in R_{0}} p_{i}\right)^{1 /\left|R_{0}\right|} \alpha^{-n} \exp \left(\frac{\alpha}{\left|R_{0}\right|} \sum_{i \in R}\left(-\tau_{i}\right)\right)
\end{aligned}
$$

and then we find the minimum of the last expression by differentiating it with respect to $\alpha$. We obtain

$$
F(\alpha) \leqq 1-\left|R_{0}\right|^{1-n}\left(\prod_{i \in R_{0}} p_{i}\right)^{1 /\left|R_{0}\right|}\left(\sum_{i \in R}\left(-\tau_{i}\right)\right)^{n}(e / n)^{n}<0
$$

for all $\alpha>0$, which implies that the condition (2.3) of Theorem 2.1 is satisfied.

The proof of the part (ii) is similar and we omit it.

REMARK 2.1. In [17], Ladas and Stavroulakis have proved that if $I=R_{0}$ then all bounded solutions of $(\mathrm{M},+1)$ with $n$ odd or $(\mathrm{M},-1)$ with $n$ even are oscillatory provided that one of the following conditions holds:

$$
\begin{array}{ll}
\left(\mathrm{a}^{\prime}\right) & p_{i}\left(-\tau_{i}\right)^{n}>(n / e)^{n} \quad \text { for some } i \in R, \\
\left(\mathrm{a}^{\prime \prime}\right) & \left(\sum_{i \in R} p_{i}\right)(-\tau)^{n}>(n / e)^{n}, \quad \text { where } \tau=\max \left\{\tau_{i}, i \in R\right\},
\end{array}
$$

and if $I=A_{0}$ then all unbounded solutions of $(\mathrm{M},-1)$ with $n$ even or $(\mathrm{M},-1)$ with $n$ odd are oscillatory provided that one of the following conditions is satisfied.

$$
\begin{aligned}
& p_{i} \tau_{i}^{n}>(n / e)^{n} \quad \text { for some } i \in A, \\
& \left(\sum_{i \in A} p_{i}\right) \tau^{n}>(n / e)^{n}, \quad \text { where } \tau=\min \left\{\tau_{i}, i \in A\right\} .
\end{aligned}
$$

Obviously, each of $\left(\mathrm{a}^{\prime}\right)$ and $\left(\mathrm{a}^{\prime \prime}\right)$ implies that our condition (a) holds and each of $\left(c^{\prime}\right)$ and $\left(c^{\prime \prime}\right)$ implies that our condition (c) holds.

REMARK 2.2. The conditions (a) and (b) (resp. (c) and (d)) are independent. In fact, for the first order inequality 


$$
\left\{x^{\prime}(t)+\frac{1}{9} x(t-1)+x\left(t-\frac{1}{9}\right)\right\} \operatorname{sgn} x(t) \leqq 0, \quad t \geqq t_{0}
$$

resp.

$$
\left\{x^{\prime}(t)-\frac{1}{9} x(t+1)-x\left(t+\frac{1}{9}\right)\right\} \operatorname{sgn} x(t) \geqq 0, \quad t \geqq t_{0},
$$

the condition (b) (resp. (d)) is satisfied, but (a) (resp. (c)) does not hold.

On the other hand, for the inequality

$$
\left\{x^{\prime}(t)+\frac{1}{8 e} x(t-1)+\frac{1}{2 e} x(t-2)\right\} \operatorname{sgn} x(t) \leqq 0, \quad t \geqq t_{0}
$$

resp.

$$
\left\{x^{\prime}(t)-\frac{1}{8 e} x(t+1)-\frac{1}{2 e} x(t+2)\right\} \operatorname{sgn} x(t) \geqq 0, \quad t \geqq t_{0}
$$

the condition (a) (resp. (c)) holds, but (b) (resp. (d)) is not satisfied.

Combining the results contained in Theorem 2.2 we have the following oscillation theorem for functional differential inequalities of mixed type of the form $(\mathrm{M}, \sigma)$.

Theorem 2.3. ( i ) Let $n$ be even. Then all solutions of the inequality $(\mathrm{M},+1)$ are oscillatory.

(ii) Let $n$ be odd. All solutions of $(\mathrm{M},+1)$ are oscillatory if $R \neq \phi$ and one of the conditions (a) and (b) is satisfied.

(iii) Let $n$ be even. All solutions of $(\mathrm{M},-1)$ are oscillatory if $R \neq \phi$, $A \neq \phi$, one of the conditions (a) and (b) and one of the conditions (c) and (d) are satisfied.

(iv) Let $n$ be odd. All solutions of (M, -1) are oscillatory if $A \neq \phi$ and one of the conditions (c) and (d) is satisfied.

The proof of this theorem can be carried out in essentially the same way as the proofs of Theorems 5.1 and 5.2 in [17], so we omit it.

REMARK 2.3. As far as we know, Theorems 2.2 and 2.3 are new. Besides their crucial role in proving our main oscillation theorems for the neutral equation $(\mathrm{E}, \sigma)$, they have also their own importance.

\section{$\S 3$. Classification of nonoscillatory solutions of neutral equation $(\mathrm{E}, \boldsymbol{\sigma})$}

Obviously, if $x(t)$ is a nonoscillatory solution of the equation $(\mathrm{E}, \sigma)$, then 
$x(t)+\lambda x(t+\rho)$ is eventually of constant sign, and so either

$$
x(t)[x(t)+\lambda x(t+\rho)]>0
$$

or

$$
x(t)[x(t)+\lambda x(t+\rho)]<0
$$

for all sufficiently large $t$. Assume first that (3.1) holds. Then the function $u(t)=x(t)+\lambda x(t+\rho)$ satisfies (2.1) for all large $t$, which implies that $u(t)$ is a function of Kiguradze degree $l$ for some $l \in\{0,1, \cdots, n\}$ with $(-1)^{n-l-1} \sigma=1$. Denote by $\mathscr{N}_{l}^{+}$the set of all solutions $x(t)$ of (E, $\sigma$ ) satisfying (3.1) and such that $x(t)+\lambda x(t+\rho)$ is of degree $l$.

Now assume that (3.2) holds. Then the function $u(t)=x(t)+\lambda x(t+\rho)$ satisfies $(-\sigma) u(t) u^{(n)}(t)<0$ for all large $t$ and so $u(t)$ is a function of Kiguradze degree $l$ for some $l \in\{0,1, \cdots, n\}$ with $(-1)^{n-l} \sigma=1$. Denote by $\mathscr{N}_{l}^{-}$the set of all solutions $x(t)$ of (E, $\sigma)$ which satisfy (3.2) and for which $x(t)+\lambda x(t+\rho)$ is of degree $l$.

We claim that all the sets $\mathscr{N}_{l}^{+}$and $\mathscr{N}_{i}^{-}, 0<l<n$, are empty. Assume first that $\lambda<0$. If $x \in \mathscr{N}_{l}^{+}$(resp. $\left.\mathscr{N}_{l}^{-}\right), 0<l<n$, then the function $u(t)=x(t)+$ $\lambda x(t+\rho)$ satisfies $|u(t)|<|x(t)|$ (resp. $(1 / \lambda)|u(t-\rho)|<|x(t)|)$ for all large $t$ and it is a nonoscillatory solution of degree $l, 0<l<n$, of the functional differential inequality

$$
\left\{\sigma u^{(n)}(t)+\sum_{i \in I} p_{i} u\left(t+\tau_{i}\right)\right\} \operatorname{sgn} u(t) \leqq 0,
$$

resp.

$$
\left\{(-\sigma) u^{(n)}(t)+\sum_{i \in I}\left(-\frac{p_{i}}{\lambda}\right) u\left(t-\rho+\tau_{i}\right)\right\} \operatorname{sgn} u(t) \leqq 0,
$$

which is impossible (see Section 2).

On the other hand, let $\lambda \geqq 0$ and assume that $x \in \mathscr{N}_{l}^{+}, 0<l<n$. (Observe that $\mathscr{N}_{l}^{-}=\phi$ for all $l=0,1, \cdots, n$ in this case.) Then the functions $u(t)=x(t)$ $+\lambda x(t+\rho)$ and $v(t)=u(t)+\lambda u(t+\rho)$ are both solutions of Eq. (E, $\sigma)$ and, moreover, for all sufficiently large $t$ we have

$$
|v(t)|=|u(t)|+\lambda|u(t+\rho)| \leqq(1+\lambda)|u(t)|
$$

provided that $\rho \leqq 0$ and

$$
|v(t)|=|u(t)|+\lambda|u(t+\rho)|<(1+\lambda)|u(t+\rho)|
$$

provided that $\rho>0$. Consequently, the function $v(t)=u(t)+\lambda u(t+\rho)$ is a nonoscillatory solution of degree $l, 0<l<n$, of the functional differential inequality 


$$
\left\{\sigma v^{(n)}(t)+\sum_{i \in I} \frac{p_{i}}{1+\lambda} v\left(t+\tau_{i}\right)\right\} \operatorname{sgn} v(t) \leqq 0
$$

if $\rho<0$ and the inequality

$$
\left\{\sigma v^{(n)}(t)+\sum_{i \in I} \frac{p_{i}}{1+\lambda} v\left(t-\rho+\tau_{i}\right)\right\} \operatorname{sgn} v(t) \leqq 0
$$

if $\rho>0$. In both cases we get a contradiction with the assertion proved in the preceding section.

Summarizing the above observations we see that the decomposition of the set $\mathscr{N}$ of all possible nonoscillatory solutions of Eq. $(\mathrm{E}, \sigma)$ reduces to

$$
\begin{array}{ll}
\mathscr{N}=\mathscr{N}_{0}^{-} \cup \mathscr{N}_{n}^{-} & \text {for }(\mathrm{E},+1) \text { with } n \text { even } \\
\mathscr{N}=\mathscr{N}_{0}^{+} \cup \mathscr{N}_{n}^{-} & \text {for }(\mathrm{E},+1) \text { with } n \text { odd } \\
\mathscr{N}=\mathscr{N}_{0}^{+} \cup \mathscr{N}_{n}^{+} & \text {for }(\mathrm{E},-1) \text { with } n \text { even } \\
\mathscr{N}=\mathscr{N}_{n}^{+} \cup \mathscr{N}_{0}^{-} & \text {for }(\mathrm{E},-1) \text { with } n \text { odd. }
\end{array}
$$

Furthermore, it is natural to expect that an additional restriction upon $\lambda$ and $\rho$ may force some of the solution classes appearing in the above classification relations to be empty. The following simple lemmas imply that this is indeed true.

LEMMA 3.1. Let $x(t)$ be a positive solution of the functional inequality

$$
x(t)+\lambda x(t+\rho)<0, \quad t \geqq t_{0},
$$

where $-1 \leqq \lambda<0$ and $\rho \neq 0$ are constants, defined on an interval $\left[t_{x}, \infty\right), t_{x} \geqq t_{0}$.

(i) Suppose that $\rho<0$. Then $x(t)$ is bounded on $\left[t_{x}, \infty\right)$. If, moreover, $-1<\lambda<0$, then $\lim _{t \rightarrow \infty} x(t)=0$.

(ii) Suppose that $\rho>0$. Then $x(t)$ is bounded away from zero on $\left[t_{x}, \infty\right)$ that is, there exists a positive constant $c$ such that $x(t) \geqq c$ on $\left[t_{x}, \infty\right)$. If, moreover, $-1<\lambda<0$, then $\lim _{t \rightarrow \infty} x(t)=\infty$.

LEMMA 3.2. Let $x(t)$ be a positive solution of the functional inequality

$$
x(t)+\lambda x(t+\rho)>0, \quad t \geqq t_{0},
$$

where $\lambda \leqq-1$ and $\rho \neq 0$ are constants, defined on an interval $\left[t_{x}, \infty\right), t_{x} \geqq t_{0}$.

(i) Suppose that $\rho>0$. Then $x(t)$ is bounded on $\left[t_{x}, \infty\right)$. If, moreover, $\lambda<-1$, then $\lim _{t \rightarrow \infty} x(t)=0$.

(ii) Suppose that $\rho<0$. Then $x(t)$ is bounded away from zero on $\left[t_{x}, \infty\right)$. If, moreover, $\lambda<-1$, then $\lim _{t \rightarrow \infty} x(t)=\infty$.

Proof of Lemma 3.1. Let $x(t)$ be a positive solution of (3.9) defined on 
$\left[t_{x}, \infty\right), t_{x} \geqq t_{0}$, and let $\rho<0$. Then

$$
x(t)<-\lambda x(t+\rho) \leqq x(t+\rho)
$$

for all large $t$, which implies that $x(t)$ is bounded. In the case $-1<\lambda<0$ we have, by iteration,

$$
x(t-n \rho)<(-\lambda)^{n} x(t) \rightarrow 0 \quad \text { as } n \rightarrow \infty,
$$

and, consequently, $\lim _{t \rightarrow \infty} x(t)=0$, which completes the proof of part (i).

Now let $\rho>0$. Then

$$
x(t+\rho) \geqq-\lambda x(t+\rho)>x(t)
$$

for $t \geqq t_{x}$, which implies that $x(t)$ has to be bounded away from zero on $\left[t_{x}, \infty\right)$. For $-1<\lambda<0$ we get, by iteration,

$$
x(t+n \rho)>\left(-\frac{1}{\lambda}\right)^{n} x(t) \rightarrow \infty \quad \text { as } \quad n \rightarrow \infty,
$$

so that $\lim _{t \rightarrow \infty} x(t)=\infty$ and the proof is complete.

The proof of Lemma 3.2 can be done in an analogous way, so we omit it.

On the basis of Lemmas 3.1 and 3.2 we get the following classification relations for the set $\mathscr{N}$ of all possible nonoscillatory solutions of Eq. (E, $\sigma$ ).

A) Let $\rho<0$. Then

$$
\begin{array}{ll}
\mathscr{N}=\phi & \text { if } \lambda \geqq 0, \\
\mathscr{N}=\mathscr{N}_{0}^{-} & \text {if }-1 \leqq \lambda<0, \\
\mathscr{N}=\mathscr{N}_{0}^{-} \cup \mathscr{N}_{n}^{-} & \text {if } \lambda<-1,
\end{array}
$$

for $(\mathrm{E},+1)$ with $n$ even,

$$
\begin{array}{ll}
\mathscr{N}=\mathscr{N}_{0}^{+} & \text {if } \lambda>-1, \\
\mathscr{N}=\phi & \text { if } \lambda=-1, \\
\mathscr{N}=\mathscr{N}_{n}^{-} & \text {if } \lambda<-1,
\end{array}
$$

for $(\mathrm{E},+1)$ with $n$ odd,

$$
\begin{array}{ll}
\mathscr{N}=\mathscr{N}_{0}^{+} \cup \mathscr{N}_{n}^{+} & \text {if } \lambda>-1, \\
\mathcal{N}=\mathscr{N}_{n}^{+} & \text {if } \lambda \leqq-1,
\end{array}
$$

for $(\mathrm{E},-1)$ with $n$ even, and

$$
\begin{array}{ll}
\mathscr{N}=\mathscr{N}_{n}^{+} & \text {if } \lambda \geqq 0, \\
\mathscr{N}=\mathscr{N}_{n}^{+} \cup \mathscr{N}_{0}^{-} & \text {if } \lambda<0,
\end{array}
$$


for $(\mathrm{E},-1)$ with $n$ odd.

B) Let $\rho>0$. Then

$$
\begin{array}{ll}
\mathscr{N}=\phi & \text { if } \lambda \geqq 0, \\
\mathscr{N}=\mathscr{N}_{n}^{-} & \text {if }-1 \leqq \lambda<0, \\
\mathscr{N}=\mathscr{N}_{0}^{-} \cup \mathscr{N}_{n}^{-} & \text {if } \lambda<-1,
\end{array}
$$

for $(\mathrm{E},+1)$ with $n$ even,

$$
\begin{array}{ll}
\mathscr{N}=\mathscr{N}_{0}^{+} & \text {if } \lambda \geqq 0, \\
\mathscr{N}=\mathscr{N}_{0}^{+} \cup \mathscr{N}_{n}^{-} & \text {if } \lambda<0,
\end{array}
$$

for $(\mathrm{E},+1)$ with $n$ odd,

$$
\begin{array}{ll}
\mathscr{N}=\mathscr{N}_{0}^{+} \cup \mathscr{N}_{n}^{+} & \text {if } \lambda>-1, \\
\mathscr{N}=\mathscr{N}_{0}^{+} & \text {if } \lambda \leqq-1,
\end{array}
$$

for $(\mathrm{E},-1)$ with $n$ even, and

$$
\begin{array}{ll}
\mathscr{N}=\mathscr{N}_{n}^{+} & \text {if } \lambda>-1, \\
\mathscr{N}=\phi & \text { if } \lambda=-1, \\
\mathscr{N}=\mathscr{N}_{0}^{-} & \text {if } \lambda<-1,
\end{array}
$$

for $(\mathrm{E},-1)$ with $n$ odd.

The results obtained in Section 2 enable us to establish conditions under which all the solution classes $\mathscr{N}_{0}^{+}, \mathscr{N}_{n}^{+}, \mathscr{N}_{0}^{-}$and $\mathscr{N}_{n}^{-}$appearing in the above classification relations are empty, that is, all solutions of Eq. (E, $\sigma$ ) have to be oscillatory. This is done in the next section.

\section{§4. Oscillation criteria for neutral equation $(\mathrm{E}, \sigma)$}

We begin by deriving "ordinary” functional differential inequalities of mixed type which must possess nonoscillatory solutions of degree 0 and/or $n$ provided that Eq. $(\mathrm{E}, \sigma)$ is assumed to have a nonoscillatory solution. Then we apply Theorem 2.3 to these inequalities and show that such a situation is impossible.

First we examine the case where $\rho<0$.

Let $x(t)$ be a nonoscillatory solution of $(\mathrm{E}, \sigma)$ such that $x \in \mathscr{N}_{0}^{+}$. If $\lambda \leqq 0$, then the function $u(t)=x(t)+\lambda x(t+\rho)$ satisfies $|u(t)| \leqq|x(t)|$ for all large, $t$, so that it is a nonoscillatory solution (of degree 0 ) of the inequality

$$
\left\{\sigma u^{(n)}(t)+\sum_{i \in I} p_{i} u\left(t+\tau_{i}\right)\right\} \operatorname{sgn} u(t) \leqq 0
$$

for all sufficiently large $t$. 
On the other hand, if $\lambda>-1$, then $u(t)$ itself is a nonoscillatory solution of Eq. (E, $\sigma)$ such that $u \in \mathscr{N}_{0}^{+}$and the function $v(t)=u(t)+\lambda u(t+\rho)$ satisfies

$$
|v(t)|<(1+\lambda)|u(t+\rho)| \text { eventually, }
$$

and, consequently, it is a nonoscillatory solution (of degree 0) of

$$
\left\{\sigma v^{(n)}(t)+\sum_{i \in I} \frac{p_{i}}{1+\lambda} v\left(t-\rho+\tau_{i}\right)\right\} \operatorname{sgn} v(t) \leqq 0
$$

for all large $t$.

Let $x(t)$ be such that $x \in \mathscr{N}_{n}^{+}$. If $\lambda \leqq 0$, then, obviously, $|u(t)| \leqq|x(t)|$ eventually and $u(t)$ is a solution of degree $n$ of the inequality (4.1). If $\lambda \geqq 0$, then the function $v(t)=u(t)+\lambda u(t+\rho)$ satisfies $|v(t)| \leqq(1+\lambda)|u(t)|$ and it is a nonoscillatory solution (of degree $n$ ) of

$$
\left\{\sigma v^{(n)}(t)+\sum_{i \in I} \frac{p_{i}}{1+\lambda} v\left(t+\tau_{i}\right)\right\} \operatorname{sgn} v(t) \leqq 0
$$

for all large $t$.

Now assume that $x(t)$ is a member of $\mathscr{N}_{0}^{-}$. (We recall that this is possible only if $\lambda<0$.) For any $\lambda<0$ we get the estimation

$$
|x(t)|>-\frac{1}{\lambda}|u(t-\rho)| \quad \text { eventually, }
$$

so that $u(t)$ is a solution (of degree 0 ) of the inequality

$$
\left\{(-\sigma) u^{(n)}(t)+\sum_{i \in I}\left(-\frac{p_{i}}{\lambda}\right) u\left(t-\rho+\tau_{i}\right)\right\} \operatorname{sgn} u(t) \leqq 0
$$

for all large $t$. If $x \in \mathscr{N}_{n}^{-}$then for any negative $\lambda$ we can similarly conclude that $u(t)$ is a solution of degree $n$ of (4.4). If, moreover, $\lambda<-1$, then for $x \in \mathscr{N}_{n}^{-}$the function $u(t)$ is from $\mathscr{N}_{n}^{-}$as well and $v(t)=u(t)+\lambda u(t+\rho)$ satisfies

$$
|u(t)|>-\frac{1}{1+\lambda}|v(t-\rho)| \text { eventually, }
$$

which implies that $v(t)$ is a solution (of degree $n$ ) of the inequality

$$
\left\{(-\sigma) v^{(n)}(t)+\sum_{i \in I}\left(-\frac{p_{i}}{1+\lambda}\right) v\left(t-\rho+\tau_{i}\right)\right\} \operatorname{sgn} v(t) \leqq 0
$$

for all sufficiently large $t$.

Applying Theorem 2.3 to the inequalities (4.1)-(4.5), we obtain the following oscillation theorems for the neutral functional differential equation (E, $\sigma$ ) in the case $\rho<0$. In addition to $R_{0}, R, A_{0}$ and $A$ defined in Section 2 
we need the following subsets of $I$ :

$$
\begin{array}{ll}
\bar{R}_{0}=\left\{i \in I: \tau_{i}-\rho \leqq 0\right\}, & \bar{R}=\left\{i \in I: \tau_{i}-\rho<0\right\}, \\
\bar{A}_{0}=\left\{i \in I: \tau_{i}-\rho \geqq 0\right\}, & \bar{A}=\left\{i \in I: \tau_{i}-\rho>0\right\} .
\end{array}
$$

THEOREm 4.1. Consider the equation $(\mathrm{E},+1)$ with $n$ even and $\rho<0$.

(i) Let $\lambda \geqq 0$. Then all solutions of $(\mathrm{E},+1)$ are oscillatory.

(ii) Let $-1 \leqq \lambda<0 . \quad$ All solutions of $(\mathrm{E},+1)$ are oscillatory if $\bar{R} \neq \phi$ and one of the following conditions is satisfied:

$$
\begin{aligned}
& \sum_{i \in \overline{\bar{R}}}\left(-\frac{p_{i}}{\lambda}\right)\left(\rho-\tau_{i}\right)^{n}>(n / e)^{n} ; \\
& \left|\bar{R}_{0}\right|^{1-n}\left(\sum_{i \in \bar{R}}\left(\rho-\tau_{i}\right)\right)^{n}\left(\prod_{i \in \bar{R}_{0}}\left(-\frac{p_{i}}{\lambda}\right)\right)^{1 /\left|\bar{R}_{0}\right|}>(n / e)^{n} .
\end{aligned}
$$

(iii) Let $\lambda<-1$. All solutions of $(\mathrm{E},+1)$ are oscillatory if, $\bar{R} \neq \phi, \bar{A} \neq \phi$, one of the conditions $\left(\mathrm{a}_{1}\right)$ and $\left(\mathrm{b}_{1}\right)$ and one of the following conditions are satisfied:

$$
\begin{aligned}
& \sum_{i \in \bar{A}}\left(-\frac{p_{i}}{1+\lambda}\right)\left(\tau_{i}-\rho\right)^{n}>(n / e)^{n} ; \\
& \left|\bar{A}_{0}\right|^{1-n}\left(\sum_{i \in \bar{A}}\left(\tau_{i}-\rho\right)\right)^{n}\left(\prod_{i \in \bar{A}_{0}}\left(-\frac{p_{i}}{1+\lambda}\right)\right)^{1 /\left|\bar{A}_{0}\right|}>(n / e)^{n} .
\end{aligned}
$$

Theorem 4.2. Consider the equation $(\mathrm{E},+1)$ with $n$ odd and $\rho<0$.

(i) Let $\lambda<-1$. All solutions of $(\mathrm{E},+1)$ are oscillatory if $\bar{A} \neq \phi$ and one of the conditions $\left(\mathrm{c}_{1}\right)$ and $\left(\mathrm{d}_{1}\right)$ is satisfied.

(ii) Let $\lambda=-1$. Then all solutions of $(\mathrm{E},+1)$ are oscillatory.

(iii) Let $\lambda>-1$. All solutions of $(\mathrm{E},+1)$ are oscillatory if $\bar{R} \neq \phi$ and one of the following conditions is satisfied:

$$
\begin{aligned}
& \sum_{i \in \overline{\bar{R}}} \frac{p_{i}}{1+\lambda}\left(\rho-\tau_{i}\right)^{n}>(n / e)^{n} ; \\
& \left|\bar{R}_{0}\right|^{1-n}\left(\sum_{i \in \bar{R}}\left(\rho-\tau_{i}\right)\right)^{n}\left(\prod_{i \in \bar{R}_{0}} \frac{p_{i}}{1+\lambda}\right)^{1 /\left|\bar{R}_{0}\right|}>(n / e)^{n} .
\end{aligned}
$$

(iv) Let $-1<\lambda<0$. All solutions of $(\mathrm{E},+1)$ are oscillatory if $R \neq \phi$ and one of the following conditions is satisfied:

$$
\begin{aligned}
& \sum_{i \in R} p_{i}\left(-\tau_{i}\right)^{n}>(n / e)^{n} ; \\
& \left|R_{0}\right|^{1-n}\left(\sum_{i \in \bar{R}}\left(-\tau_{i}\right)\right)^{n}\left(\prod_{i \in R_{0}} p_{i}\right)^{1 /\left|R_{0}\right|}>(n / e)^{n} .
\end{aligned}
$$

TheOREM 4.3. Consider the equation $(\mathrm{E},-1)$ with $n$ even and $\rho<0$. 
(i) Let $\lambda \leqq-1$. All solutions of $(\mathrm{E},-1)$ are oscillatory if $A \neq \phi$ and one of the following conditions is satisfied:

$$
\begin{aligned}
& \sum_{i \in A} p_{i} \tau_{i}^{n}>(n / e)^{n} ; \\
& \left|A_{0}\right|^{1-n}\left(\sum_{i \in A} \tau_{i}\right)^{n}\left(\prod_{i \in A_{0}} p_{i}\right)^{1 /\left|A_{0}\right|}>(n / e)^{n} .
\end{aligned}
$$

(ii) Let $-1<\lambda<0$. All solutions of $(\mathrm{E},-1)$ are oscillatory if $A \neq \phi$, one of the conditions $\left(\mathrm{c}_{2}\right)$ and $\left(\mathrm{d}_{2}\right)$ is satisfied and either $\bar{R} \neq \phi$ and one of the conditions $\left(\mathrm{a}_{2}\right)$ and $\left(\mathrm{b}_{2}\right)$ holds or $R \neq \phi$ and one of the conditions $\left(\mathrm{a}_{3}\right)$ and $\left(\mathrm{b}_{3}\right)$ holds.

(iii) Let $\lambda \geqq 0$. All solutions of $(\mathrm{E},-1)$ are oscillatory if $\bar{R} \neq \phi, A \neq \phi$, one of the conditions $\left(\mathrm{a}_{2}\right)$ and $\left(\mathrm{b}_{2}\right)$ and one of the following conditions hold:

$$
\begin{aligned}
& \sum_{i \in A} \frac{p_{i}}{1+\lambda} \tau_{i}^{n}>(n / e)^{n} ; \\
& \left|A_{0}\right|^{1-n}\left(\sum_{i \in A} \tau_{i}\right)^{n}\left(\prod_{i \in A_{0}} \frac{p_{i}}{1+\lambda}\right)^{1 /\left|A_{0}\right|}>(n / e)^{n} .
\end{aligned}
$$

Theorem 4.4. Consider the equation $(\mathrm{E},-1)$ with $n$ odd and $\rho<0$.

(i ) Let $\lambda \geqq 0$. All solutions of $(\mathrm{E},-1)$ are oscillatory if $A \neq \phi$ and one of the conditions $\left(\mathrm{c}_{3}\right)$ and $\left(\mathrm{d}_{3}\right)$ is satisfied.

(ii) Let $\lambda<0$. All solutions of $(E,-1)$ are oscillatory if $\bar{R} \neq \phi, A \neq \phi$ and, moreover, one of the conditions $\left(\mathrm{a}_{1}\right)$ and $\left(\mathrm{b}_{1}\right)$ and one of the conditions $\left(\mathrm{c}_{2}\right)$ and $\left(\mathrm{d}_{2}\right)$ are satisfied.

Now the proofs of the above theorems follow.

Proof of Theorem 4.1. (i ) If $\lambda \geqq 0$, then $\mathscr{N}=\phi$ for $(\mathrm{E},+1)$ with $n$ even, so that all solutions are oscillatory.

(i) If $-1 \leqq \lambda<0$, then $\mathscr{N}_{0}^{-}$is the only possible class of nonoscillatory solutions of $(\mathrm{E},+1)$. Suppose that $\mathscr{N}_{0}^{-} \neq \phi$. Then the inequality (4.4) has a nonoscillatory solution of degree 0 . However, the condition $\left(a_{1}\right)\left(\right.$ or $\left.\left(b_{1}\right)\right)$ implies that this is impossible.

(iii) If $\lambda<-1$, then $\mathscr{N}=\mathscr{N}_{0}^{-} \cup \mathscr{N}_{n}^{-}$. Assuming $\mathscr{N}_{0}^{-} \neq \phi$ we again conclude that (4.4) must possess a nonoscillatory solution (of degree 0 ) which contradicts the assumption that one of the conditions $\left(a_{1}\right)$ and $\left(b_{1}\right)$ is satisfied. Similarly, $\mathscr{N}_{n}^{-} \neq \phi$ implies the existence of a nonoscillatory solution of degree $n$ of the inequality (4.5) which is a contradiction to the assumption that $\left(c_{1}\right)$ or $\left(\mathrm{d}_{1}\right)$ holds.

Proof of Theorem 4.2. (i) In the case $\lambda<-1$ the set $\mathscr{N}$ of all possible nonoscillatory solutions of $(\mathrm{E},+1)$ with $n$ odd reduces to $\mathscr{N}_{n}$. If 
$\mathscr{N}_{n}^{-} \neq \phi$, then the inequality (4.5) possesses a nonoscillatory solution of degree $n$. However, this is a contradiction because from $\left(c_{1}\right)\left(\right.$ or $\left.\left(d_{1}\right)\right)$ and Theorem 2.3 (iv) it follows that (4.5) cannot have any nonoscillatory solution.

(ii) If $\lambda=-1$, then the set $\mathscr{N}$ for $(\mathrm{E},+1)$ with $n$ odd is necessarily empty and so all solutions of $(\mathrm{E},+1)$ must oscillate.

(iii) If $\lambda>-1$, then $\mathscr{N}=\mathscr{N}_{0}^{+}$. Assuming $\mathscr{N}_{0}^{+} \neq \phi$ we easily see that (4.2) has a solution of degree 0 , which leads to a contradiction with Theorem 2.3 (ii) applied to the inequality (4.2).

(iv) Let $-1<\lambda<0$. If $\mathscr{N}_{0}^{+} \neq \phi$, then (4.1) must possess a nonoscillatory solution of degree 0. However, Theorem 2.3 (ii) applied to (4.1) shows, in view of $\left(a_{3}\right)$ (or $\left.\left(b_{3}\right)\right)$, that (4.1) cannot have nonoscillatory solutions. Consequently, $\mathscr{N}_{0}^{+}=\phi$ and the proof is complete since in this case $\mathscr{N}_{0}^{+}$is the only possible nonoscillatory solution class.

Proof of Theorem 4.3. (i ) In the case $\lambda \leqq-1$ we have $\mathscr{N}=\mathscr{N}_{n}^{+}$for $(\mathrm{E},-1)$ with $n$ even. If $\mathscr{N}_{n}^{+} \neq \phi$, then the inequality (4.1) possesses a nonoscillatory solution of degree $n$. However, the condition $\left(c_{2}\right)\left(\right.$ or $\left.\left(d_{2}\right)\right)$ prevents (4.1) from having nonoscillatory solutions of this kind. Thus we conclude that $\mathscr{N}_{n}^{+}=\phi$.

(ii) If $-1<\lambda<0$, then $\mathscr{N}=\mathscr{N}_{0}^{+} \cup \mathscr{N}_{n}^{+}$. As in the proof of part (i) we see that $\mathscr{N}_{n}^{+}=\phi$. On the other hand, if $\mathscr{N}_{0}^{+} \neq \phi$, then the inequalities (4.1) and (4.2) have nonoscillatory solutions of degree 0 , which contradicts $\left(\mathrm{a}_{2}\right)$ (or $\left(\mathrm{b}_{2}\right)$ ) in case $\bar{R} \neq \phi$ and $\left(\mathrm{a}_{3}\right)$ (or $\left(\mathrm{b}_{3}\right)$ ) in the case $R \neq \phi$.

(iii) If $\lambda \geqq 0$, then $\mathscr{N}=\mathscr{N}_{0}^{+} \cup \mathscr{N}_{n}^{+}$. Suppose that $\mathscr{N}_{0}^{+}$has a member. Then the inequality (4.2) admits a nonoscillatory solution of degree 0 , which is impossible since the condition $\left(\mathrm{a}_{2}\right)$ (or $\left(\mathrm{b}_{2}\right)$ ) implies the nonexistence of nonoscillatory solutions of this kind for (4.2). Similarly, if $\mathscr{N}_{n}^{+} \neq \phi$, then (4.3) has a solution of order $n$, which contradicts $\left(c_{3}\right)\left(\right.$ or $\left.\left(d_{3}\right)\right)$.

Proof of Theorem 4.4. (i ) In the case $\lambda \geqq 0$, the set of all possible nonoscillatory solutions of $(\mathrm{E},-1)$ with $n$ odd reduces to $\mathscr{N}_{n}^{+}$. If $\mathscr{N}_{n}^{+} \neq \phi$, then (4.3) possesses a nonoscillatory solution of degree $n$, which is a contradiction because from $\left(c_{3}\right)$ (or $\left(d_{3}\right)$ ) and Theorem 2.3 (iv) it follows that (4.3) cannot have nonoscillatory solutions.

(ii) If $\lambda<0$, then $\mathscr{N}_{n}^{+}$and $\mathscr{N}_{0}^{-}$are the only possible classes of nonoscillatory solutions of $(\mathrm{E},-1)$. However, under conditions of the theorem both $\mathscr{N}_{n}^{+}$and $\mathscr{N}_{0}^{-}$have to be empty. In fact, if $\mathscr{N}_{n}^{+} \neq \phi$, then (4.1) has a nonoscillatory solution (of degree $n$ ), which contradicts Theorem 2.3 (iv) and if $\mathscr{N}_{0}^{-}$ $\neq \phi$, then (4.4) has a nonoscillatory solution (of degree 0 ), which contradicts Theorem 2.3 (ii). The proof is complete.

Let us now turn to the case where $\rho>0$. 
If $x(t)$ is a nonoscillatory solution of $(\mathrm{E}, \sigma)$ such that $x \in \mathscr{N}_{0}^{+}$, then for all large $t$ we have $|u(t)| \leqq|x(t)|$ provided that $\lambda \leqq 0$ and $|v(t)| \leqq(1+\lambda)|u(t)|$ provided that $\lambda \geqq 0$, where the functions $u(t)$ and $v(t)$ are defined as before. Consequently, $u(t)$ (resp. $v(t))$ is a nonoscillatory solution of degree 0 of (4.1) (resp. (4.3)).

If $x(t)$ is a member of $\mathscr{N}_{n}^{+}$, then the function $u(t)$ satisfies $|u(t)| \leqq|x(t)|$ for all large $t$ provided that $\lambda \leqq 0$ and the function $v(t)$ satisfies $|v(t)| \leqq(1+\lambda)$ $\cdot|u(t+\rho)|$ eventually provided that $\lambda>-1$. It follows that $u(t)$ (resp. $v(t))$ is a nonoscillatory solution (of degree $n$ ) of (4.1) (resp. (4.2)).

Next assume that $x \in \mathscr{N}_{0}^{-}$. Then for all large $t$

$$
|x(t)|>-\frac{1}{\lambda}|u(t-\rho)|
$$

provided that $\lambda<0$ and

$$
|v(t)|<-(1+\lambda)|u(t+\rho)|
$$

provided that $\lambda<-1$. Consequently, if $\lambda<0$ then $u(t)$ is a nonoscillatory solutino (of degree 0 ) of (4.4) and if $\lambda<-1$ then $v(t)$ is a nonoscillatory solution (of degree 0 ) of (4.5).

Finally, if $\lambda<0$ and if $x \in \mathscr{N}_{n}^{-}$, then $|x(t)|>-(1 / \lambda)|u(t-\rho)|$ for all large $t$, so that the function $u(t)$ is a nonoscillatory solution (of degree $n$ ) of the inequality (4.4).

These observations enable us to establish the following oscillation results for Eq. (E, $\sigma$ ) in the case $\rho>0$.

Theorem 4.5. Consider the equation $(\mathrm{E},+1)$ with $n$ even and $\rho>0$.

(i) Let $\lambda \geqq 0$. Then all solutions of $(\mathrm{E},+1)$ are oscillatory.

(ii) Let $-1 \leqq \lambda<0$. All solutions of $(\mathrm{E},+1)$ are oscillatory if $\bar{A} \neq \phi$ and one of the following conditions is satisfied:

$$
\begin{aligned}
& \sum_{i \in \bar{A}}\left(-\frac{p_{i}}{\lambda}\right)\left(\tau_{i}-\rho\right)^{n}>(n / e)^{n} ; \\
& \left|\bar{A}_{0}\right|^{1-n}\left(\sum_{i \in \bar{A}}\left(\tau_{i}-\rho\right)\right)^{n}\left(\prod_{i \in \bar{A}_{0}}\left(-\frac{p_{i}}{\lambda}\right)\right)^{1 /\left|\bar{I}_{0}\right|}>(n / e)^{n} .
\end{aligned}
$$

(iii) Let $\lambda<-1$. All solutions of $(\mathrm{E},+1)$ are oscillatory if $\bar{R} \neq \phi, \bar{A} \neq \phi$, one of the conditions $\left(\mathrm{c}_{4}\right)$ and $\left(\mathrm{d}_{4}\right)$ and one of the following conditions are satisfied:

$$
\begin{aligned}
& \sum_{i \in \overline{\bar{R}}}\left(-\frac{p_{i}}{1+\lambda}\right)\left(\rho-\tau_{i}\right)^{n}>(n / e)^{n} ; \\
& \left|\bar{R}_{0}\right|^{1-n}\left(\sum_{i \in \bar{E}}\left(\rho-\tau_{i}\right)\right)^{n}\left(\prod_{i \in \bar{R}_{0}}\left(-\frac{p_{i}}{1+\lambda}\right)\right)^{1 /\left|\bar{R}_{0}\right|}>(n / e)^{n} .
\end{aligned}
$$


THEOREm 4.6. Consider the equation $(\mathrm{E},+1)$ with $n$ odd and $\rho>0$.

(i ) Let $\lambda \geqq 0$. All solutions of $(\mathrm{E},+1)$ are oscillatory if $R \neq \phi$ and one of the following conditions is satisfied:

$$
\begin{aligned}
& \sum_{i \in R} \frac{p_{i}}{1+\lambda}\left(-\tau_{i}\right)^{n}>(n / e)^{n} \\
& \left|R_{0}\right|^{1-n}\left(\sum_{i \in R}\left(-\tau_{i}\right)\right)^{n}\left(\prod_{i \in R_{0}} \frac{p_{i}}{1+\lambda}\right)^{1 /\left|R_{0}\right|}>(n / e)^{n} .
\end{aligned}
$$

(ii) Let $\lambda<0$. All solutions of $(\mathrm{E},+1)$ are oscillatory if $R \neq \phi, \bar{A} \neq \phi$, one of the conditions $\left(\mathrm{a}_{3}\right)$ and $\left(\mathrm{b}_{3}\right)$ and one of the conditions $\left(\mathrm{c}_{4}\right)$ and $\left(\mathrm{d}_{4}\right)$ are satisfied.

TheORem 4.7. Consider the equation $(\mathrm{E},-1)$ with $n$ even and $\rho>0$.

(i) Let $\lambda \leqq-1$. All solutions of $(\mathrm{E},-\mathrm{i})$ are oscillatory if $R \neq \phi$ and one of the conditions $\left(\mathrm{a}_{3}\right)$ and $\left(b_{3}\right)$ is satisfied.

(ii) Let $-1<\lambda<0$. All solutions of $(\mathrm{E},-1)$ are oscillatory if $R \neq \phi$, one of the conditions $\left(\mathrm{a}_{3}\right)$ and $\left(\mathrm{b}_{3}\right)$ is satisfied and either $A \neq \phi$ and one of the conditions $\left(\mathrm{c}_{2}\right)$ and $\left(\mathrm{d}_{2}\right)$ holds or $\bar{A} \neq \phi$ and one of the following conditions is satisfied:

$$
\begin{aligned}
& \sum_{i \in \bar{A}} \frac{p_{i}}{1+\lambda}\left(\tau_{i}-\rho\right)^{n}>(n / e)^{n} \\
& \left|\bar{A}_{0}\right|^{1-n}\left(\sum_{i \in \bar{A}}\left(\tau_{i}-\rho\right)\right)^{n}\left(\prod_{i \in \bar{A}_{0}} \frac{p_{i}}{1+\lambda}\right)^{1 /\left|\bar{A}_{0}\right|}>(n / e)^{n} .
\end{aligned}
$$

(iii) Let $\lambda \geqq 0$. All solutions of $(\mathrm{E},-1)$ are oscillatory if $R \neq \phi, \bar{A} \neq \phi$, one of the conditions $\left(\mathrm{a}_{5}\right)$ and $\left(\mathrm{b}_{5}\right)$ and one of the conditions $\left(\mathrm{c}_{5}\right)$ and $\left(\mathrm{d}_{5}\right)$ are satisfied.

THEOREM 4.8. Consider the equation $(\mathrm{E},-1)$ with $n$ odd and $\rho>0$.

(i) Let $\lambda<-1$. All solutions of $(\mathrm{E},-1)$ are oscillatory if $\bar{R} \neq \phi$ and one of the conditions $\left(\mathrm{a}_{4}\right)$ and $\left(\mathrm{b}_{4}\right)$ is satisfied.

(ii) Let $\lambda=-1$. Then all solutions of $(\mathrm{E},-1)$ are oscillatory.

(iii) Let $\lambda>-1$. All solutions of $(\mathrm{E},-1)$ are oscillatory if $\bar{A}=\phi$ and one of the conditions $\left(\mathrm{c}_{5}\right)$ and $\left(\mathrm{d}_{5}\right)$ is satisfied.

(iv) Let $-1<\lambda<0$. All solutions of $(\mathrm{E},-1)$ are oscillatory if $A \neq \phi$ and one of the conditions $\left(\mathrm{c}_{2}\right)$ and $\left(\mathrm{d}_{2}\right)$ is satisfied.

The proofs of Theorems 4.5-4.8 are very similar to those of Theorems 4.1-4.4, so we omit them. 


\section{References}

[1] T. A. Čanturia, On specific criteria of oscillation of solutions of linear differential equations with retarded argument, Ukrain. Mat. Ž., 38 (1986), 662-665. (Russian)

[2] M. K. Grammatikopoulos, E. A. Grove and G. Ladas, Oscillations of first order neutral delay differential equations, J. Math. Anal. Appl., 120 (1986), 510-520.

[ 3 ] M. K. Grammatikopoulos, E. A. Grove and G. Ladas, Oscillation and asymptotic behavior of neutral differential equations with deviating arguments, Applicable Anal., 22 (1986), 1-19.

[4] M. K. Grammatikopoulos, E. A. Grove and G. Ladas, Oscillation and asymptotic behavior of second order neutral differential equations with deviating arguments, Canadian Mathematical Society Proceedings, 8 (1987), 153-161.

[5] M. K. Grammatikopoulos, G. Ladas and A. Meimaridou, Oscillation of second order neutral delay differential equations, Radovi Mat., 1 (1985), 267-274.

[6] M. K. Grammatikopoulos, G. Ladas and Y. G. Sficas, Oscillation and asymptotic behavior of neutral equations with variable coefficients, Radovi Mat., 2 (1986), 279-303.

[ 7 ] E. A. Grove, M. R. S. Kulenović and G. Ladas, Sufficient conditions for oscillation and nonoscillation of neutral equations, J. Differential Equations, 68 (1987), $373-382$.

[8] E. A. Grove, G. Ladas and A. Meimaridou, A necessary and sufficient condition for the oscillation of neutral equations, J. Math. Anal. Appl., 126 (1987), $341-354$.

[9] A. F. Ivanov and T. Kusano, On oscillation of solutions of a class of functional differential equations, Ukrain. Mat. ̌̌., 39 (1987), 717-721, (Russian).

[10] J. Jaroš and T. Kusano, Oscillation theory of higher order linear functional differential equations of neutral type, Hiroshima Math. J., 18 (1988), 509-531.

[11] I. T. Kiguradze, On the oscillation of solutions of the equation $d^{m} u / d t^{m}+a(t)|u|^{n}$ sign $u=0$, Mat. Sb., 65(107) (1964), 172-187. (Russian)

[12] M. R. S. Kulenović, G. Ladas and A. Meimaridou, Necessary and sufficient condition for oscillations of neutral differential equations, J. Austral. Math. Soc. Ser. B, 28 (1987), 362-375.

[13] T. Kusano, On even order functional differential equations with advanced and retarded arguments, J. Differential Equations, 45 (1982), 75-84.

[14] G. Ladas and Y. G. Sficas, Oscillations of neutral delay differential equations, Canad. Math. Bull., 29(4) (1986), 438-445.

[15] G. Ladas and Y. G. Sficas, Oscillations of higher-order neutral equations, J. Austral. Math. Soc. Ser. B, 27 (1986), 502-511.

[16] G. Ladas, Y. G. Sficas and I. P. Stavroulakis, Necessary and sufficient conditions for oscillations of higher order delay differential equations, Trans. Amer. Math. Soc., 285 (1984), 81-90.

[17] G. Ladas and I. P. Stavroulakis, Oscillations of differential equations of mixed type, J. Mathematical and Physical Sci., 18 (1984), 254-262.

[18] Ch. G. Philos, On the existence of nonoscillatory solutions tending to zero at $\infty$ for differential equations with positive delays, Arch. Math., 36 (1981), 168-178.

[19] J. Ruan, Oscillations of neutral differential difference equations with several retarded arguments, Sci. Sinica Ser. A, 19 (1986), 1132-1144.

[20] Y. G. Sficas and I. P. Stavroulakis, Necessary and sufficient conditions for oscillations of neutral differential equations, J. Math. Anal. Appl., 123 (1987), 494-507. 
[21] A. I. Zahariev and D. D. Bainov, Oscillating properties of the solutions of a class of neutral type functional differential equations, Bull. Austral. Math. Soc., 22 (1980), 365-372.

[22] A. I. Zahariev and D. D. Bainov, On some oscillation criteria for a class of neutral type functional differential equations, J. Austral. Math. Soc. Ser. B, 28 (1986), 229-239.

Jaroslav JAROŠ

Department of Mathematical ANALYsis

Faculty of Mathematics and Physics

KOMENSKY UNIVERSITY

Bratislava, Czechoslovakia

Takaŝi Kusano

Department of Mathematics

FACULTY OF SCIENCE

HiRoshima UNIVERSITY

HIROSHIMA, JAPAN 